\title{
False positive latex tests negative by ELISA for toxoplasma IgG
}

\author{
G M SUTEHALL, T G WREGHITT Clinical Microbiology and Public Health Laboratory, \\ Addenbrooke's Hospital, Cambridge
}

SUMMARY An enzyme linked immunosorbent assay (ELISA) kit for detecting IgG class antibody to $T$ gondii was compared with the latex agglutination test to determine the specificity as a screening method in 12 patients who had undergone heart transplantation (recrudescence of $T$ gondii infection $\mathrm{n}=3$, donor acquired infection $\mathrm{n}=3$; acute cytomegalovirus infection $\mathrm{n}=6$ ). The latex agglutination test detected antibodies to primary $T$ gondii infection much earlier in the infection than the ELISA, but the ELISA method was useful for detecting previous infection.

It is concluded that the ELISA technique is more complex to perform than the latex agglutination test but the use of IgM and IgG assays combined could reduce the number of samples sent to the reference laboratory and thus reduce the time taken to obtain a final result.

It has been reported that a latex agglutination test for detecting antibodies to Toxoplasma gondii (Toxoreagent, Mast Diagnostics), which is widely used as a screening test for toxoplasmosis, can give rise to false positive results with serum samples from heart transplant patients with cytomegalovirus (CMV) infection, which seems to be IgM-mediated.' A more recent report shows that false positive results have been found with serum samples from people who are not specifically immunocompromised but who also have CMV infection. ${ }^{2}$ We examined an enzyme linked immunosorbent assay (ELISA) kit for detecting IgG class antibody to $T$ gondii to determine whether it was a more specific screening test than the latex agglutination test.

\section{Material and methods}

Sequential serum samples from heart transplant patients were examined by means of the CAPTIA Toxo-G kit (Mercia Diagnostics, Guildford). Three patients who had antibodies to $T$ gondii positive at the time of transplantation and who subsequently experienced recrudescence of $T$ gondii after transplantation, three patients with primary donor-acquired $T$ gondii infection, and six cases with acute CMV infection in which the toxoplasma latex agglutination test was falsely reactive were studied. All samples were initially tested by the latex agglutination test and the reactive

Accepted for publication 1 September 1988 specimens were further tested in the dye test, haemagglutination test, and $\mu$-capture IgM ELISA test at the Public Health Laboratory Service Toxoplasma Reference Laboratory at Leeds.

\section{Results}

For the cases with recrudescence of $T$ gondii, we found a good correlation between the latex agglutination test titre and the CAPTIA Toxo-G index (figure).

Samples with false reactivity in the latex agglutination test were not positive in the CAPTIA Toxo-G assay (figure) and neither were samples negative in the latex agglutination test.

In one case of primary toxoplasmosis, however, the ELISA did not detect antibody early in the infection and in a second case the ELISA result was only just positive, at $5 \%$ over the cut off value at the start of the antibody response, and was not strongly positive until much later. By contrast, the latex agglutination test detected $T$ gondii antibody early in the infection.

\section{Discussion}

The CAPTIA Toxo-G assay is useful for detecting previous toxoplasma infection and would be useful as a screening test in this context. The assay is not subject to the type of non-specific reactions encountered when using the Toxoreagent latex agglutination test. This could be because false positive reactions in the latex agglutination test are associated with an IgMmediated mechanism' to which the ELISA is not 
False positive latex tests negative by ELISA for toxoplasma IgG

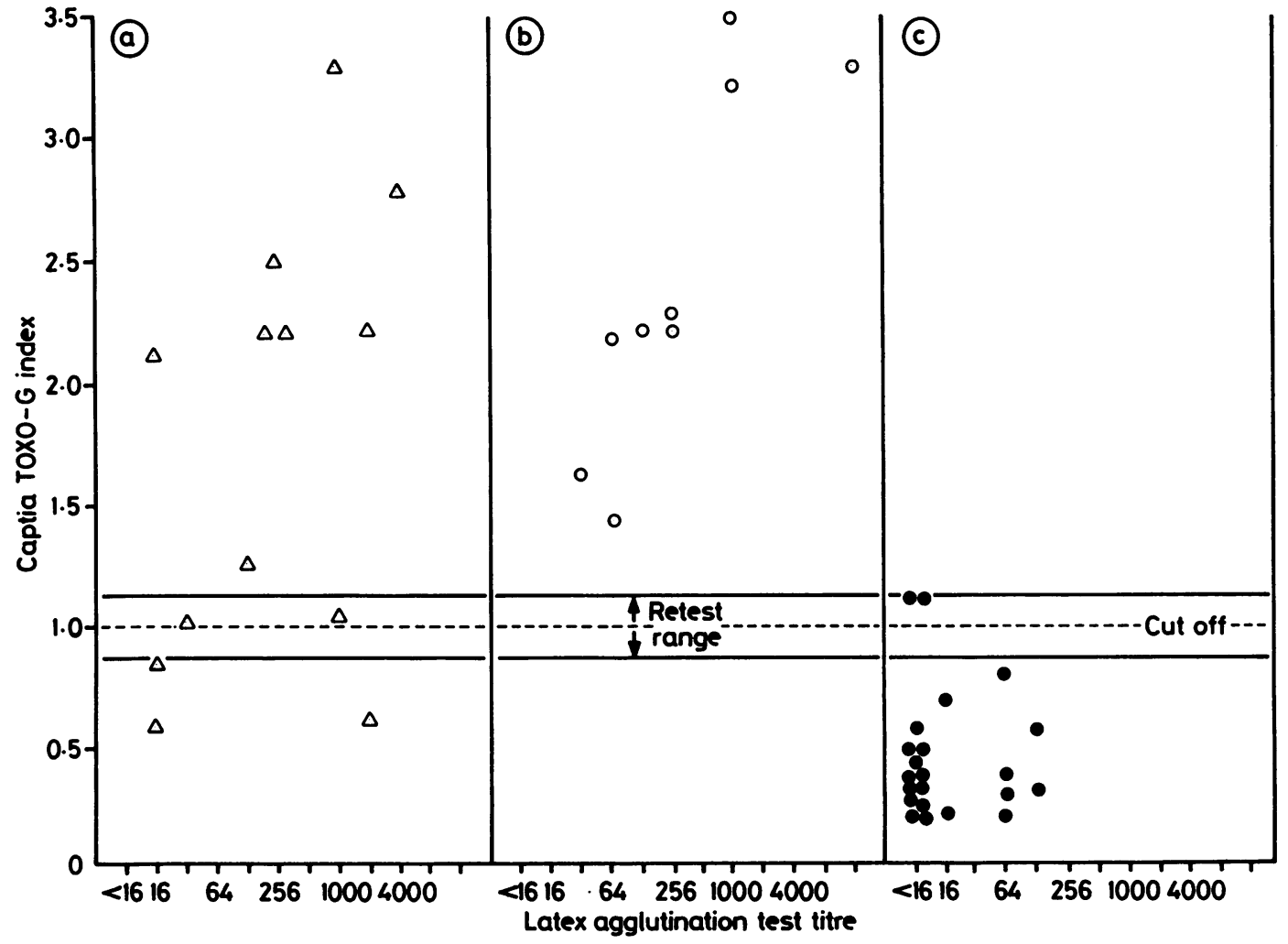

Figure $T$ gondii ELISA index and latex agglutination test titres of serum samples from heart transplant recipients: (a) primary $T$ gondii infections; (b) reactivations of $T$ gondii infection; and (c) latex agglutination test false positive samples.

susceptible. For use as a test to monitor transplant patients for donor-acquired toxoplasmosis, however, this ELISA would need to be used in conjunction with a suitable assay for detecting $T$ gondii specific IgM.

The ELISA technique is more complex to perform than that of the latex agglutination test but the use of IgM and IgG assays combined could reduce the number of samples to be sent to the reference laboratory and hence the time taken to obtain a final result. This should be taken into account when selecting the most suitable type of screening assay to use.

\section{References}

1 Wreghitt TG, Gray JJ, Balfour AH. Problems with serological diagnosis of Toxoplasma gondii infections in heart transplant patients. J Clin Pathol 1986;39:1135-9.

2 Balfour AH, Prestage ES, Noel I, Wreghitt TG. False positive results in the Toxoreagent test for Toxoplasma gondii in immunocompetent patients. J Clin Pathol 1988;41:1135-6.

Requests for reprints to: $\mathrm{Mr}$ G M Sutehall, Clinical Microbiology and Public Health Laboratory, Level 6, Addenbrooke's Hospital, Hills Road, Cambridge CB2 2QW, England. 